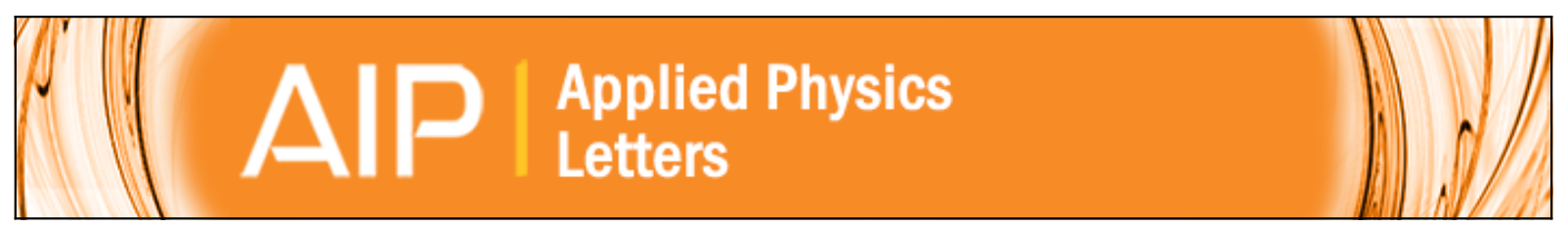

\title{
Thermal effects on transverse domain wall dynamics in magnetic nanowires
}

J. Leliaert, B. Van de Wiele, J. Vandermeulen, A. Coene, A. Vansteenkiste, L. Laurson, G. Durin, B. Van Waeyenberge, and L. Dupré

Citation: Applied Physics Letters 106, 202401 (2015); doi: 10.1063/1.4921421

View online: http://dx.doi.org/10.1063/1.4921421

View Table of Contents: http://scitation.aip.org/content/aip/journal/apl/106/20?ver=pdfcov

Published by the AIP Publishing

\section{Articles you may be interested in}

Current-induced motion of a transverse magnetic domain wall in the presence of spin Hall effect

Appl. Phys. Lett. 101, 022405 (2012); 10.1063/1.4733674

Current-induced coupled domain wall motions in a two-nanowire system

Appl. Phys. Lett. 99, 152501 (2011); 10.1063/1.3650706

Magnetic imaging of the pinning mechanism of asymmetric transverse domain walls in ferromagnetic nanowires Appl. Phys. Lett. 97, 233102 (2010); 10.1063/1.3523351

Magnetic domain wall propagation in nanowires under transverse magnetic fields

J. Appl. Phys. 103, 073906 (2008); 10.1063/1.2887918

Simulated domain wall dynamics in magnetic nanowires

J. Appl. Phys. 99, 08G107 (2006); 10.1063/1.2176890
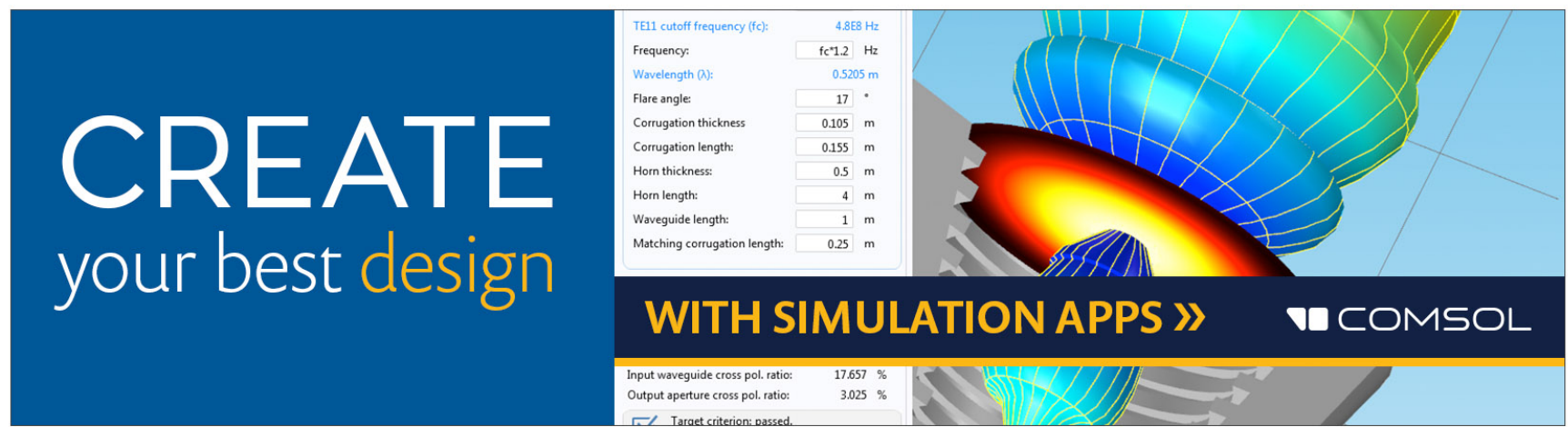


\title{
Thermal effects on transverse domain wall dynamics in magnetic nanowires
}

\author{
J. Leliaert, ${ }^{1,2, a)}$ B. Van de Wiele, ${ }^{1}$ J. Vandermeulen, ${ }^{1}$ A. Coene, ${ }^{1}$ A. Vansteenkiste ${ }^{2}$ \\ L. Laurson, ${ }^{3}$ G. Durin, ${ }^{4,5}$ B. Van Waeyenberge, ${ }^{2}$ and L. Dupré ${ }^{1}$ \\ ${ }^{1}$ Department of Electrical Energy, Systems and Automation, Ghent University, 9000 Gent, Belgium \\ ${ }^{2}$ Department of Solid State Sciences, Ghent University, 9000 Gent, Belgium \\ ${ }^{3}$ COMP Centre of Excellence and Helsinki Institute of Physics, Department of Applied Physics, \\ Aalto University School of Science, P.O. Box 11100, FI-00076 Aalto, Finland \\ ${ }^{4}$ Istituto Nazionale di Ricerca Metrologica, Strada delle Cacce 91, 10135 Torino, Italy \\ ${ }^{5}$ ISI Foundation, Via Alassio 11/c, 10126 Torino, Italy
}

(Received 26 February 2015; accepted 9 May 2015; published online 18 May 2015)

\begin{abstract}
Magnetic domain walls are proposed as data carriers in future spintronic devices, whose reliability depends on a complete understanding of the domain wall motion. Applications based on an accurate positioning of domain walls are inevitably influenced by thermal fluctuations. In this letter, we present a micromagnetic study of the thermal effects on this motion. As spin-polarized currents are the most used driving mechanism for domain walls, we have included this in our analysis. Our results show that at finite temperatures, the domain wall velocity has a drift and diffusion component, which are in excellent agreement with the theoretical values obtained from a generalized 1D model. The drift and diffusion component are independent of each other in perfect nanowires, and the mean square displacement scales linearly with time and temperature. (C) 2015 AIP Publishing LLC. [http://dx.doi.org/10.1063/1.4921421]
\end{abstract}

Several future spintronic devices ${ }^{1,2}$ rely on the ability to accurately manipulate the position of magnetic domain walls in nanowires. For instance, in the racetrack memory, ${ }^{1}$ data are stored in magnetic domains separated by domain walls moved by spin polarized currents. ${ }^{3,4}$ Recently, new schemes have been proposed in which the domain walls themselves represent the data bits. ${ }^{5}$ However, domain walls are subject to thermal fluctuations which can compromise the position of the walls, and thus the integrity of the data. Consequently, thermal effects should be taken into account in the design and should first be understood. In micromagnetic simulations, thermal fluctuations are either included by a jump-noise process $^{6,7}$ or a stochastic field ${ }^{8}$ as determined by Brown. ${ }^{9}$ Using this approach, the influence of thermal fluctuations at high driving forces has been investigated. ${ }^{10}$ It was found that the effect of thermal fluctuations is negligible in the flow regime. However, most experiments are performed at low velocities, because of the high current densities (of the order of $1 \mathrm{~A} / \mu \mathrm{m}^{2}$ ) required to move domain walls.

In this letter, we investigate the effects of finite temperatures on transverse domain wall motion in the absence of magnetic defects such as grain boundaries. We find that thermal fluctuations result in a diffusive motion of the domain wall independent of the excitation current. These results are understood within the framework of a generalized 1D model ${ }^{11}$ where we incorporated temperature and get an analytical expression for the diffusion, in excellent agreement with numerical simulations. This easy inclusion of thermal effects is important for future work where this approach can be applied to the more realistic case of nanowires with imperfections.

On the microscopic level, magnetization dynamics is described by the Landau-Lifshitz-Gilbert equation ${ }^{12}$ extended

\footnotetext{
${ }^{a)}$ Electronic mail: jonathan.leliaert@ugent.be
}

with two spin transfer torque terms ${ }^{13}$ due to the current density $\mathbf{J}$,

$$
\begin{aligned}
\frac{\partial \mathbf{m}}{\partial t}= & -\gamma \mathbf{m} \times \mathbf{H}_{\mathrm{eff}}+\alpha \mathbf{m} \times \frac{\partial \mathbf{m}}{\partial t} \\
& -[b \mathbf{J} \cdot \nabla] \mathbf{m}+\beta \mathbf{m} \times[b \mathbf{J} \cdot \nabla] \mathbf{m} .
\end{aligned}
$$

In this equation, $\gamma$ depicts the gyromagnetic ratio, $\alpha$ the Gilbert damping constant, $b=P \mu_{B} / e M_{s}\left(1+\beta^{2}\right)$ with $P$ the polarization of the spin-polarized current, $e$ the electron charge, $\mu_{B}$ the Bohr magneton, $\beta$ the degree of non-adiabaticity, ${ }^{14,15}$ and $M_{s}$ the saturation magnetization. $\mathbf{m}$, the normalized magnetization (with unit length), and $\mathbf{H}_{\text {eff }}$, the effective field, are both space and time varying vector fields. The effective field consists of different terms: ${ }^{16}$ the external field, the demagnetizing field, and fields due to the exchange interaction and the anisotropy of the material. Thermal fluctuations are taken into account by a stochastic thermal field, ${ }^{9}$ $\mathbf{H}_{\mathrm{th}}$, contributing to the effective field. This thermal field [Eq. (2)] is uncorrelated in space and time, and has a magnitude determined by the fluctuation dissipation theorem, ${ }^{9,10}$

$$
\begin{aligned}
\left\langle\mathbf{H}_{\mathrm{th}}\right\rangle_{\text {stat }} & =0 \\
\left\langle\mathbf{H}_{\mathrm{th}, \mathrm{i}}(t) \mathbf{H}_{\mathrm{th}, \mathrm{j}}\left(t^{\prime}\right)\right\rangle_{\text {stat }} & =q \delta_{d}\left(t-t^{\prime}\right) \delta_{d, i j} \\
q & =\frac{2 k_{B} T \alpha}{M_{s} \gamma \mu_{0} V} .
\end{aligned}
$$

The operator \langle\rangle$_{\text {stat }}$ indicates a statistical average over different realizations, indices $i, j$, and $k$ represent the axes in a cartesian system, $\delta_{d}$ is the Dirac delta function, $k_{B}$ the Boltzmann constant, $T$ the temperature, $\mu_{0}$ the vacuum permeability, and $V$ the volume on which the thermal field is calculated, which in our simulations is the size of the finite difference cells. We performed our simulations using the software package MuMax3. ${ }^{17}$ To simulate domain wall motion in an infinite wire, we restrict 
the computational region to an $800 \mathrm{~nm}$ wide window centered around the moving domain wall. ${ }^{4}$ Magnetic charges at the window edges are compensated. The cross-section $S$ of the wire is $100 \times 10 \mathrm{~nm}^{2}$.

To analyze the motion of the domain wall, we employ a generalization $^{11}$ of the $1 \mathrm{D}$ model. ${ }^{18-20}$ We have extended this model to include the effects of externally applied fields in any direction, combined with a spin-polarized current. The resulting formulas are shown in Eqs. (3) and (4), where \langle\rangle represents a spatial average over the computational region $R$. In the derivation of the generalized 1D model, all terms which are asymmetric in the magnetization are collected in one contribution $\mathcal{O}$ (asymmetric), which is equal to 0 for symmetric domain walls ${ }^{11}$

$$
\begin{aligned}
\dot{x}\left(H_{\mathrm{ext}}, J_{x}\right)= & \frac{L_{x} \gamma\langle\delta\rangle}{2 \alpha} H_{\mathrm{ext}, \mathrm{x}}-\frac{\beta}{\alpha} b J_{x} \\
& -\frac{L_{x}}{2 \alpha}\left\langle\delta \frac{\partial \phi}{\partial t}\right\rangle+\mathcal{O} \text { (asymmetric) } \\
\left\langle\delta \frac{\partial \phi}{\partial t}\right\rangle\left(H_{\mathrm{ext}}, J_{x}\right)= & \frac{\gamma}{1+\alpha^{2}} H_{\mathrm{ext}, \mathrm{x}}\langle\delta\rangle-\frac{2}{L_{x}} \frac{\beta-\alpha}{1+\alpha^{2}} b J_{x} \\
& +\frac{\alpha \gamma}{1+\alpha^{2}}\left(H_{\mathrm{ext}, \mathrm{z}}\left\langle m_{y}\right\rangle-H_{\mathrm{ext}, \mathrm{y}}\left\langle m_{z}\right\rangle\right) \\
& -\frac{\alpha \gamma}{1+\alpha^{2}} \frac{M_{s}}{\langle\sqrt{\delta}\rangle^{2}}\left(N_{z}-N_{y}\right)\left\langle m_{y}\right\rangle\left\langle m_{z}\right\rangle \\
& +\mathcal{O}(\text { asymmetric }) .
\end{aligned}
$$

Here, $L_{x}$ is the width of the computational window, $N_{y}$ and $N_{z}$ are effective demagnetizing factors, while the angle $\phi$ is the out-of-plane tilting of the domain wall. Furthermore, $\langle\delta\rangle$ is the fraction of $R$ which is not magnetized along the nanowire

$$
\langle\delta\rangle=\frac{\left\langle m_{y}^{2}+m_{z}^{2}\right\rangle}{\left\langle m_{x}^{2}+m_{y}^{2}+m_{z}^{2}\right\rangle}=\left\langle m_{y}^{2}+m_{z}^{2}\right\rangle .
$$

Hence, $L_{x}\langle\delta\rangle$ can be interpreted as a measure of the domain wall width. Equation (3) shows that in general, the domain wall velocity is not only determined by the direct action of a driving field or current but is also affected by the time variation of the magnetization tilting $\partial \phi / \partial t$ and the asymmetry of the domain wall. Below the Walker breakdown, the domain wall tilting is fixed, resulting in a vanishing third term of Eq. (3).

To isolate the effects of temperature, we performed a first set of simulations, applying only a spin-polarized current and assuming perfect adiabaticity $(\beta=0)$. Typical material parameters for Permalloy ${ }^{21}$ were used. In the absence of temperature, no net motion of the domain wall is expected under the Walker breakdown ${ }^{3,15}$ as is clear from Eq. (3). Hence, all steady state domain wall dynamics can be attributed to thermal effects. On the transverse domain wall, shown in Fig. 1(a), we have applied a current density $J=1 \mathrm{~A} / \mu \mathrm{m}^{2}$ at $300 \mathrm{~K}$ for 100 ns. In Fig. 1(b), 1000 paths of the domain wall simulated with different realizations of the temperature are shown. The red line highlights one typical path. On the right side of Fig. 1(b), the distribution of the final domain wall positions after $100 \mathrm{~ns}$ is shown. Remarkably, the combined action of (a) z

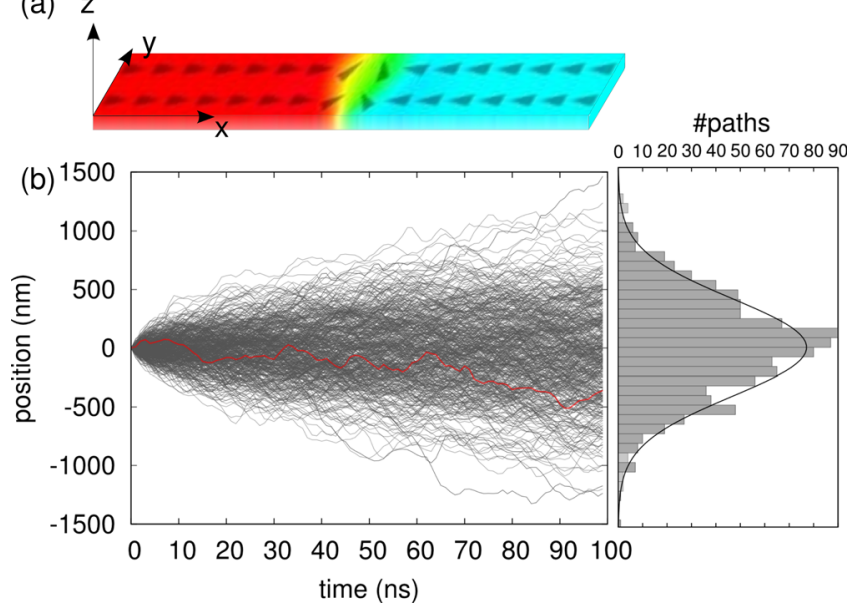

FIG. 1. (a) The transverse domain wall positioned in the center of the computational region. The colors and arrows represent the direction of the magnetization. Note the absence of closure domains at the outer edges due to charge compensation. The lengths of the wire in the direction indicated by the axes in the coordinate system are 800,100 , and $10 \mathrm{~nm}$, respectively. (b) The left side of the figure shows the positions as function of time for 1000 domain walls in a magnetic nanowire at $300 \mathrm{~K}$, and $J=1 A / \mu m^{2}(\beta=0)$. One randomly chosen path is highlighted in red. The right side shows the distribution of their final positions, with a fitted Gaussian curve centered at 0 , proving that the motion is diffusion with no average displacement.

thermal fluctuations and current does not give rise to an average domain wall motion: the data are described by a Gaussian with zero average. Consequently, the motion can be interpreted as a random walk resulting in diffusion without net motion and characterized by a mean square displacement (MSD). To investigate the influence of current on the diffusion, we performed similar simulations varying $J$ from 0 to $10 \mathrm{~A} / \mathrm{\mu m}^{2}$ (close to the Walker breakdown current density of $\pm 14 \mathrm{~A} / \mu^{2} \mathrm{~m}^{2}$. For each current density, 500 realizations of the temperature are simulated. The results are shown in Fig. 2(a), where the red bullets represent the MSD divided by time, as this quantity is independent of the simulation time. As a function of current density, almost constant values of MSD/t are found, indicating that-apart from the indirect effect due to a decrease in $L_{x}\langle\delta\rangle$ for higher current densities (as shown in Fig. 3(e)) - the current density does not influence the diffusion characteristics. In the nanowire, the domain wall tilts out of plane until it reaches an offset tilting determined by the current density. Here, at zero temperature, all torques cancel out and the wall does not move. ${ }^{3}$ Thermal fluctuations give rise to an additional field torque responsible for the motion of the domain wall. These thermal fluctuations are independent of the current density and thus give rise to similar diffusion. Repeating these simulations at different temperatures shows that MSD/t scales linearly with temperature [see Fig. 2(c)] as expected in a system described by normal diffusion. ${ }^{22}$ We aim to quantitatively describe this motion. To this end, we interpret our results within the framework of a generalized 1D model. Similar to the micromagnetic approach, we add temperature to the $1 \mathrm{D}$ model as a fluctuating thermal field with properties given by Eq. (2). Now, $V$ is the volume of the domain wall.

Figure 3 clarifies the combined action of a constant field along the nanowire and a current on the domain wall at $0 \mathrm{~K}$ and with $\beta=0$. The velocity of the domain wall increases 

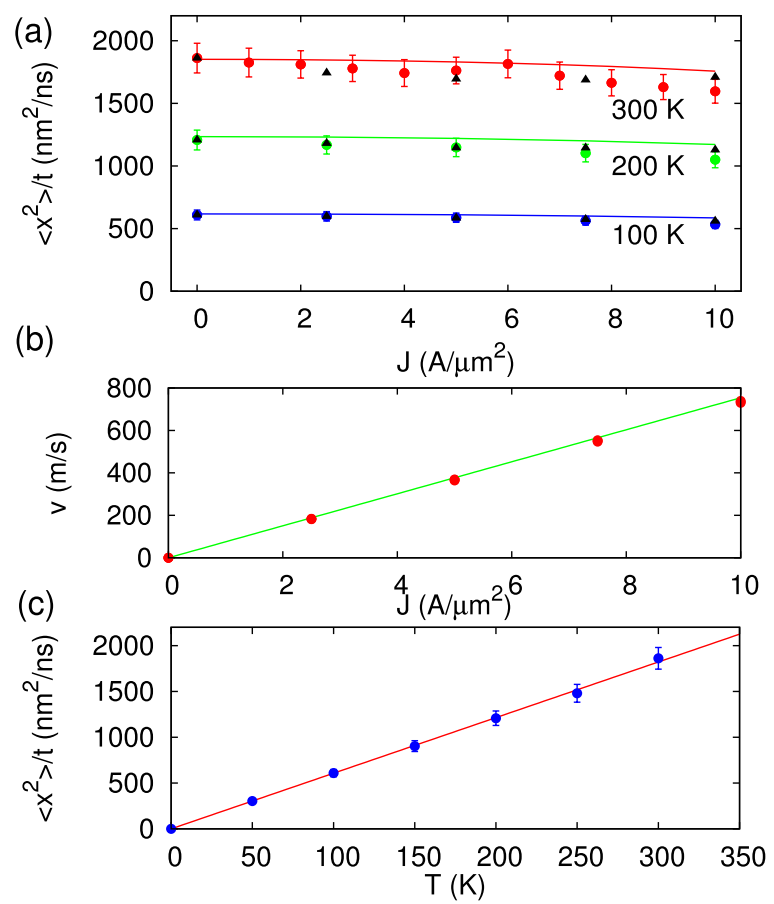

FIG. 2. (a) The domain walls' mean square displacement over time averaged each time over 500 realizations as function of current density at 3 different temperatures. The coloured dots with error bars show the simulated data with $\beta=0$, while the full lines show the theoretical curves expected by Eq. (7). The black triangles show the mean square displacement corrected for the drift velocity for simulations with $\beta=2 \alpha$. The error bars on these values are comparable to their counterparts for $\beta=0$ but are not shown for clarity. (b) The drift velocity of current driven domain walls with $\beta=2 \alpha$ at different temperatures. The datapoints (overlapping each other for all temperatures) are in almost perfect agreement with the theoretically expected velocities from Eq. (3), represented by the full line. (c) The mean square displacement over time as function of temperature at $J=0 \mathrm{~A} / \mu \mathrm{m}^{2}$. The full line is a fit to the data and shows that there is a linear temperature dependence.

with the increase in field strengths up to the Walker breakdown, see Fig. 3(a). Based on Eq. (3), we expect for a rigid, symmetric domain wall a linear dependence of the domain wall velocity on the external field. However, the domain wall width $L_{x}\langle\delta\rangle$ decreases with larger fields as shown in Fig. 3(b). Introducing this field dependence in Eq. (3) leads to the dotted lines in Fig. 3(a), showing that the reduction in domain wall width alone cannot explain the reduction in velocity. Indeed, the asymmetry of the wall significantly influences the dynamics. In Fig. 3(c), $L_{x}\left\langle m_{x} \delta\right\rangle$ versus applied field is shown. This quantity represents the net magnetization component along the nanowire axis within the domain wall, and is a measure for the domain wall asymmetry as it is zero for a symmetric domain wall and large for an asymmetric one. Panel(c) clearly shows that the wall gets increasingly asymmetric for larger fields. To take this asymmetry into account, we assume a linear dependence on the field [see Fig. 3(c)] with a slope $\sim \chi$, which can be interpreted as a susceptibility along the $x$-axis. This allows us to include the asymmetry in Eq. (3) by defining an effective $\langle\delta\rangle$,

$$
\left\langle\delta_{\text {eff }}\right\rangle \equiv\langle\delta\rangle(1-\chi) \approx 0.74\langle\delta\rangle .
$$

This value accomodates for the difference between the dotted lines and simulation data in Fig. 3(a). In other models, ${ }^{18-20,23}$ a similar rescaling is done by adopting different definitions of the domain wall width or by using the width as a fitting parameter, implicitly taking the asymmetry into account. In the following, we remove $\mathcal{O}$ (asymmetric) from the equations as these effects are now included in $\left\langle\delta_{\text {eff }}\right\rangle$.

Figures 3(d)-3(f) show similar simulation results, now with varying $J(\beta=0)$. In panel (d), the offset in velocity at $J=0 A / \mu m^{2}$ is determined by the applied field and $\left\langle\delta_{\text {eff }}\right\rangle$ at this field. With the increase in current density, the domain wall velocity gradually goes down. This is explained by the reduction in domain wall width for increasing current, as shown in panel (e). Panel (f) shows that currents have no influence on the domain wall asymmetry. Remarkably, the curves in panel (d) show similar behaviour as the simulation results in Fig. 2(a), which suggests that the latter can indeed be described by the combined action of a thermal field and the driving current. However, unlike the static field, the thermal field does not give rise to a drift velocity but only to diffusion.

Now, we will introduce thermal effects in the 1D model to explain our observations. Contrary to the fields used in Fig. 3, the thermal field acting on the domain wall fluctuates in time, and has no preferential direction. The spread on the domain wall positions ${ }^{22}$ is described by the MSD $=\left\langle\left(\int_{0}^{t} \dot{x} d t^{\prime}\right)^{2}\right\rangle_{\text {stat }}$, which can be quantified using Eq. (3),

$$
\begin{aligned}
\left\langle\left(\int_{0}^{t} \dot{x} d t^{\prime}\right)^{2}\right\rangle_{\text {stat }} & =\left\langle\left(\int_{0}^{t} \frac{L_{x} \gamma\left\langle\delta_{\text {eff }}\right\rangle}{2 \alpha} H_{\text {ext }, x} d t^{\prime}\right)^{2}\right\rangle_{\text {stat }} \\
& =\left\langle\frac{L_{x}^{2} \gamma^{2}\left\langle\delta_{\text {eff }}\right\rangle^{2}}{4 \alpha^{2}}\left(\int_{0}^{t} H_{t h, x} d t^{\prime}\right)^{2}\right\rangle_{\text {stat }} \\
& =\frac{L_{x}^{2} \gamma^{2}\left\langle\delta_{\text {eff }}\right\rangle^{2}}{4 \alpha^{2}} q t=\frac{\gamma k_{B} L_{x}\left\langle\delta_{\text {eff }}\right\rangle}{2 \alpha M_{s} \mu_{0} S} T t .
\end{aligned}
$$

Here, we assumed that the domain wall tilting follows the thermal fluctations sufficiently fast to neglect $\left\langle\delta_{\text {eff }} \frac{\partial \phi}{\partial t}\right\rangle_{\text {stat }}$. Furthermore, we made use of the properties in Eq. (2) and $V=\left\langle\delta_{\text {eff }}\right\rangle L_{x} S$, the volume of the domain wall. Note that this volume is dependent on the current density [cf. Fig. 2(e)]. As expected, the MSD grows linearly in time. The full lines in Fig. 2(a) show the MSD/t resulting from Eq. (7). For experimentally relevant (low) current densities, there is an almost perfect agreement between theory and simulation. Furthermore, Eq. (7) predicts that the MSD is linearly dependent on temperature, which is also confirmed by our simulations. Moreover, the model takes the small domain wall deformations into account via $\left\langle\delta_{\text {eff }}\right\rangle$. These result in the nonlinear deviations for large current densities also found in the simulations shown in Fig. 3. The remaining slight difference between the full lines and the data points is explained by the fact that we assumed a linear scaling of the asymmetry with the externally applied field, while Fig. 3(c) shows that this approximation is only valid for small fields. Also, $\left\langle\delta_{\text {eff }} \frac{\partial \phi}{\partial t}\right\rangle_{\text {stat }}$ might have a small contribution in Eq. (7).

In a last set of simulations, we applied a spin polarized current, assuming non-adiabaticity $(\beta=2 \alpha)$, and investigated the domain wall motion at different temperatures, again considering 500 realizations per datapoint. When $\beta \neq 0$, we expect a net velocity of the wall for any $J>0 A / \mu m^{2}$. In Fig. 2(b), the full line shows the domain wall velocity at $0 \mathrm{~K}$ described by 

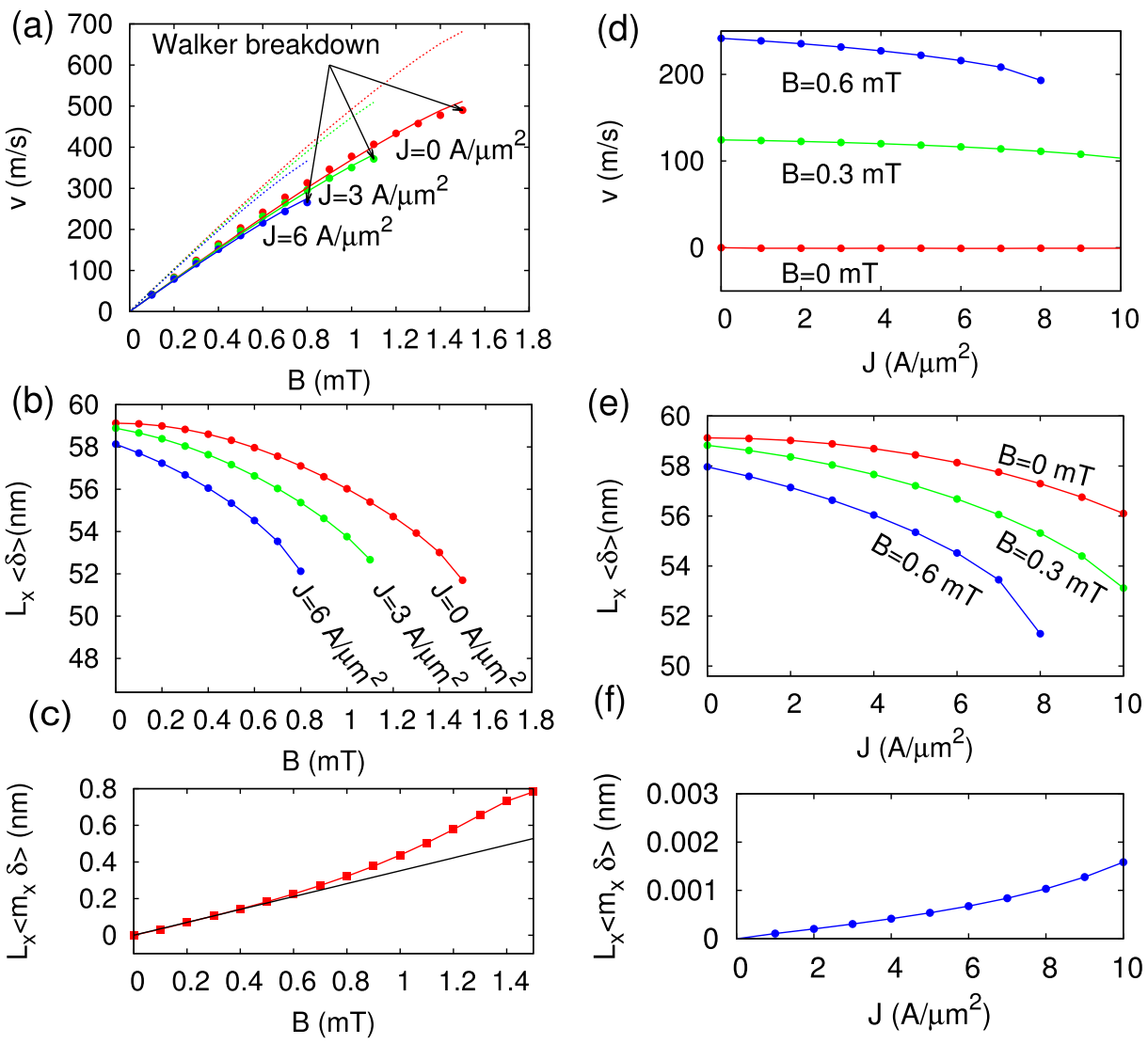

FIG. 3. Field and current driven domain wall motion at zero temperature. Both driving forces are applied along the nanowire. (a) The velocity as function of field strength for different current densities. The datapoints show the micromagnetic results, while the lines show the theoretically expected values from Eq. (3) with (full lines) and without (dotted lines) taking the asymmetry into account. (d) presents the data from (a) in a different way, to clarify that the velocity of the nanowire slightly goes down as function of current density at a fixed field strength. (b) and (e) $L_{x}\langle\delta\rangle$, a measure for the width of the domain wall. Increasing fields and current densities decrease the size of the domain wall. (c) and (f) show the asymmetry of the domain wall as function of field and current density (note the difference in scales). Current driven domain walls remain symmetric up to the Walker breakdown while field driven domain walls become more asymmetric with increasing field strengths. The full black line illustrates that the asymmetry scales linear with field at low field strengths with a slope of $0.352 \mathrm{~nm} / \mathrm{mT}$.

Eq. (3), while the datapoints depict the average domain wall velocity at $\mathrm{T}=100,200$, and $300 \mathrm{~K}$. The fact that all data coincide confirms that the drift velocity of the domain wall is unaffected by temperature. ${ }^{10}$

Additionally, at nonzero temperatures, the domain wall motion has a diffusion component. The MSD/t (corrected for the drift velocity) is shown as black triangles in Fig. 2(a), indicating that the adiabatic and non-adiabatic systems exhibit identical diffusion properties. Hence, we can conclude that the diffusion is solely determined by the domain wall shape and the temperature [cf. Eq. (7)] and is not affected by the drift velocity.

To summarize, we have investigated the influence of temperature on transverse domain wall dynamics in magnetic nanowires. Temperature is included in the micromagnetic simulations and the generalized $1 \mathrm{D}$ model as a randomly fluctuating field acting on the discretization cell and domain wall volume, respectively. In general, the domain wall motion contains a drift and a diffusion component. We verified that the drift velocity of the domain wall is unaffected by temperature and found that the domain wall diffusion gives rise to a mean square domain wall displacement which grows linearly with time. The diffusion is solely determined by temperature and the domain wall shape, which changes at higher currents. The domain wall drift and diffusion do not influence each other and can be quantitatively predicted by a generalized 1D model. Further research may focus on thermally activated domain wall motion in disordered media, i.e., the creep regime. ${ }^{24}$ At low driving forces, material defects or grain boundaries ${ }^{4}$ are potential wells $^{25}$ which act as pinning centers for the domain wall. The escape rate from these centers due to thermal fluctuations will determine the creep velocity of the domain wall through the nanowires. ${ }^{26}$

This work was supported by Flanders Research Foundation (B.V.d.W. and A.V.), Agency for Innovation by Science and Technology (I.W.T., J.V.), Ugent research fund (B.O.F. Project Nos. 01N04910 (B.V.W.) and 01J16113 (J.L. and J.V.)) and by the Academy of Finland (L.L., Projects Nos. 268302 and 251748).

${ }^{1}$ S. P. Parkin, M. Hayashi, and L. Thomas, "Magnetic domain-wall racetrack memory," Science 320(5873), 190-194 (2008).

${ }^{2}$ D. A. Allwood, G. Xiong, C. C. Faulkner, D. Atkinson, D. Petit, and R. P. Cowburn, "Magnetic domain-wall logic," Science 309, 1688-1692 (2005). ${ }^{3}$ O. Boulle, G. Malinowski, and M. Kläui, "Current-induced domain wall motion in nanoscale ferromagnetic elements," Mater. Sci. Eng. R 72(9), $159-187$ (2011).

${ }^{4}$ J. Leliaert, B. Van de Wiele, A. Vansteenkiste, L. Laurson, G. Durin, L. Dupré, and B. Van Waeyenberge, "Current-driven domain wall mobility 
in polycrystalline permalloy nanowires: A numerical study," J. Appl. Phys. 115(23), 233903 (2014).

${ }^{5}$ K. A. Omari and T. J. Hayward, "Chirality-based vortex domain-wall logic gates,” Phys. Rev. Appl. 2, 044001 (2014).

${ }^{6}$ I. Mayergoyz, G. Bertotti, and C. Serpico, "Landau-lifshitz magnetization dynamics driven by a random jump-noise process (invited)," J. Appl. Phys. 109(7), 07D312 (2011).

${ }^{7}$ I. Mayergoyz, G. Bertotti, C. Serpico, Z. Liu, and A. Lee, "Random magnetization dynamics at elevated temperatures," J. Appl. Phys. 111(7), 07D501 (2012).

${ }^{8}$ A. Lyberatos, D. V. Berkov, and R. W. Chantrell, "A method for the numerical simulation of the thermal magnetization fluctuations in micromagnetics," J. Phys.: Condens. Matter 5(47), 8911 (1993).

${ }^{9}$ W. F. Brown, "Thermal fluctuations of a single-domain particle," Phys. Rev. 130, 1677-1686 (1963).

${ }^{10}$ E. Martinez, L. Lopez-Diaz, L. Torres, C. Tristan, and O. Alejos, "Thermal effects in domain wall motion: Micromagnetic simulations and analytical model," Phys. Rev. B 75, 174409 (2007).

${ }^{11} \mathrm{~J}$. Vandermeulen, B. Van de Wiele, A. Vansteenkiste, B. Van Waeyenberge, and L. Dupré, "A collective coordinate approach to describe magnetic domain wall dynamics applied to nanowires with high perpendicular anisotropy," J. Phys. D: Appl. Phys. 48(3), 035001 (2015).

${ }^{12}$ L. D. Landau and E. M. Lifshitz, "Theory of the dispersion of magnetic permeability in ferromagnetic bodies," Phys. Z. Sowietunion 8, 153 (1935).

${ }^{13} \mathrm{~S}$. Zhang and Z. Li, "Roles of nonequilibrium conduction electrons on the magnetization dynamics of ferromagnets," Phys. Rev. Lett. 93(12), 127204 (2004).

${ }^{14}$ C. Burrowes, A. P. Mihai, D. Ravelosona, J.-V. Kim, C. Chappert, L. Vila, A. Marty, Y. Samson, F. Garcia-Sanchez, L. D. Buda-Prejbeanu, I. Tudosa, E. E. Fullerton, and J.-P. Attane, "Non-adiabatic spin-torques in narrow magnetic domain walls," Nat. Phys. 6(1), 17-21 (2010).

${ }^{15}$ J. Leliaert, B. Van de Wiele, A. Vansteenkiste, L. Laurson, G. Durin, L. Dupré, and B. Van Waeyenberge, "Influence of material defects on current-driven vortex domain wall mobility," Phys. Rev. B 89, 064419 (2014).

${ }^{16}$ W. F. Brown, Micromagnetics (Interscience Publishers, New York, 1963), p. 18.

${ }^{17}$ A. Vansteenkiste, J. Leliaert, M. Dvornik, M. Helsen, F. Garcia-Sanchez, and B. Van Waeyenberge, "The design and verification of mumax3," AIP Adv. 4(10), 107133 (2014).

${ }^{18}$ A. Thiaville, Y. Nakatani, J. Miltat, and N. Vernier, "Domain wall motion by spin-polarized current: A micromagnetic study," J. Appl. Phys. 95(11), 7049-7051 (2004).

${ }^{19}$ A. Thiaville, Y. Nakatani, J. Miltat, and Y. Suzuki, "Micromagnetic understanding of current-driven domain wall motion in patterned nanowires," Europhys. Lett. 69, 990-996 (2005).

${ }^{20}$ A. Thiaville and Y. Nakatani, "Domain-wall dynamics in nanowires and nanostrips," in Spin Dynamics in Confined Magnetic Structures III, Topics in Applied Physics Vol. 101 (Springer, Berlin-Heidelberg, 2006), pp. 161-205.

${ }^{21}$ Spin polarisation 0.56 , saturation magnetisation $860000 \mathrm{~A} / \mathrm{m}$, exchange stiffness $13 \times 10^{-12} \mathrm{~J} / \mathrm{m}$, and Gilbert damping constant $\alpha=0.01$. The nanowire was discretized in finite difference cells of size $3.125 \times 3.125$ $\times 10 \mathrm{~nm}^{3}$.

${ }^{22} \mathrm{~A}$. Einstein, Investigations on the Theory of the Brownian Movement (Courier Dover Publications, 1956).

${ }^{23}$ A. Mougin, M. Cormier, J. P. Adam, P. J. Metaxas, and J. Ferré, "Domain wall mobility, stability and walker breakdown in magnetic nanowires," Europhys. Lett. 78(5), 57007 (2007).

${ }^{24}$ J. Ferré, P. J. Metaxas, A. Mougin, J.-P. Jamet, J. Gorchon, and V. Jeudy, "Universal magnetic domain wall dynamics in the presence of weak disorder," C. R. Phys. 14(8), 651-666 (2013).

${ }^{25}$ J. Leliaert, B. Van de Wiele, A. Vansteenkiste, L. Laurson, G. Durin, L. Dupré, and B. Van Waeyenberge, "A numerical approach to incorporate intrinsic material defects in micromagnetic simulations," J. Appl. Phys. 115(17), 17D102 (2014).

${ }^{26}$ J. Ryu, S.-B. Choe, and H.-W. Lee, "Magnetic domain-wall motion in a nanowire: Depinning and creep,” Phys. Rev. B 84, 075469 (2011). 